Article

\title{
An Isocaloric Nordic Diet Modulates RELA and TNFRSF1A Gene Expression in Peripheral Blood Mononuclear Cells in Individuals with Metabolic Syndrome-A SYSDIET Sub-Study
}

\author{
Stine M. Ulven ${ }^{1, *}$, Kirsten B. Holven ${ }^{1,2}{ }^{\oplus}$, Amanda Rundblad ${ }^{1}$, Mari C. W. Myhrstad ${ }^{3}$,
} Lena Leder ${ }^{4}$, Ingrid Dahlman ${ }^{5}$, Vanessa D. de Mello ${ }^{6}{ }^{\mathbb{D}}$, Ursula Schwab ${ }^{6,7}$, Carsten Carlberg ${ }^{8} \mathbb{D}$, Jussi Pihlajamäki ${ }^{6,7}$, Kjeld Hermansen ${ }^{9}$, Lars O. Dragsted ${ }^{10}{ }^{\circledR}$, Ingibjörg Gunnarsdottir ${ }^{11}$, Lieselotte Cloetens ${ }^{12} \odot$, Björn Åkesson ${ }^{12,13}$, Fredrik Rosqvist ${ }^{14}$, Janne Hukkanen ${ }^{15}$, Karl-Heinz Herzig ${ }^{16,17}$, , Markku J Savolainen ${ }^{15}$, Ulf Risérus ${ }^{14}$, Inga Thorsdottir ${ }^{11}$, Kaisa S Poutanen ${ }^{6,18}$, Peter Arner ${ }^{5}$, Matti Uusitupa ${ }^{6}{ }^{\circ}$ and Marjukka Kolehmainen ${ }^{6}$

1 Department of Nutrition, Institute for Basic Medical Sciences, University of Oslo, 0317 Oslo, Norway; k.b.holven@medisin.uio.no (K.B.H.); Amanda.rundblad@medisin.uio.no (A.R.)

2 National Advisory Unit for Familial Hypercholesterlemia, Department of Endocrinology, Morbid Obesity and Preventive Medicine, Oslo University Hospital, 0424 Oslo, Norway

3 Department of Nursing and Health Promotion, Faculty of Health Sciences, OsloMet-Oslo Metropolitan University, 0130 Oslo, Norway; mmyhrsta@oslomet.no

4 Mills AS, Sofienberggt. 19, 0558 Oslo, Norway; lena.leder@mills.no

5 Department of Medicine (H7), Karolinska Institute, 17176 Stockholm, Sweden; Ingrid.dahlman@ki.se (I.D.); peter.arner@ki.se (P.A.)

6 School of Medicine, Institute of Public Health and Clinical Nutrition, University of Eastern Finland, 70211 Kuopio, Finland; vanessa.laaksonen@uef.fi (V.D.d.M.); ursula.schwab@uef.fi (U.S.); Jussi.Pihlajamaki@uef.fi (J.P.); Kaisa.Poutanen@vtt.fi (K.S.P.); matti.uusitupa@uef.fi (M.U.); marjukka.kolehmainen@uef.fi (M.K.)

7 Department of Medicine, Endocrinology and Clinical Nutrition, Kuopio University Hospital, 70029 Kuopio, Finland

8 Institute of Biomedicine, University of Eastern Finland, 70211 Kuopio, Finland; carsten.carlberg@uef.fi

9 Department of Endocrinology and Internal Medicine, Department of Clinical Medicine, Aarhus University Hospital, Aarhus University, 8200 Aarhus, Denmark; kjeld.hermansen@aarhus.rm.dk

10 Department of Nutrition, Exercise and Sports, Faculty of Science, University of Copenhagen, 2200 Copenhagen, Denmark; ldra@nexs.ku.dk

11 Unit for Nutrition Research, University of Iceland and Landspitali-The National University Hospital of Iceland, 101 Reykjavík, Iceland; ingigun@hi.is (I.G.); ingathor@hi.is (I.T.)

12 Biomedical Nutrition, Pure and Applied Biochemistry, Lund University, 22100 Lund, Sweden; Lieselotte.cloetens@tbiokem.lth.se (L.C.); bjorn.akesson@tbiokem.lth.se (B.Å.)

13 Department of Clinical Nutrition, Skåne University Hospital, 22100 Lund, Sweden

14 Department of Public Health and Caring Sciences, Clinical Nutrition and Metabolism, Uppsala University, 75122 Uppsala, Sweden; Fredrik.rosqvist@pubcare.uu.se (F.R.); ulf.riserus@pubcare.uu.se (U.R.)

15 Institute of Clinical Medicine, Department of Internal Medicine and Biocenter Oulu, University of Oulu, Medical Research Center, Oulu University Hospital, 90220 Oulu, Finland; janne.hukkanen@oulu.fi (J.H.); markku.savolainen@oulu.fi (M.J.S.)

16 Institute of Biomedicine, Biocenter of Oulu, Medical Research Center, Faculty of Medicine, University of Oulu, and Oulu University Hospital, 90220 Oulu, Finland; karl-heinz.herzig@oulu.fi

17 Department of Gastroenterology and Metabolism, Poznan University of Medical Sciences, 60572 Poznan, Poland

18 VTT Technical Research Centre of Finland, 021100 Espoo, Finland

* Correspondence: smulven@medisin.uio.no; Tel.: +47-22840208 


\begin{abstract}
A healthy dietary pattern is associated with a lower risk of metabolic syndrome (MetS) and reduced inflammation. To explore this at the molecular level, we investigated the effect of a Nordic diet (ND) on changes in the gene expression profiles of inflammatory and lipid-related genes in peripheral blood mononuclear cells (PBMCs) of individuals with MetS. We hypothesized that the intake of an ND compared to a control diet (CD) would alter the expression of inflammatory genes and genes involved in lipid metabolism. The individuals with MetS underwent an 18/24-week randomized intervention to compare a ND with a CD. Eighty-eight participants (66\% women) were included in this sub-study of the larger SYSDIET study. Fasting PBMCs were collected before and after the intervention and changes in gene expression levels were measured using TaqMan Array Micro Fluidic Cards. Forty-eight pre-determined inflammatory and lipid related gene transcripts were analyzed. The expression level of the gene tumor necrosis factor (TNF) receptor superfamily member $1 \mathrm{~A}$ (TNFRSF1A) was down-regulated $(p=0.004)$, whereas the nuclear factor kappa-light-chain-enhancer of activated B cells (NF- $\mathrm{KB}$ ) subunit, RELA proto-oncogene, was up-regulated $(p=0.016)$ in the ND group compared to the $\mathrm{CD}$ group. In conclusion, intake of an ND in individuals with the MetS may affect immune function.
\end{abstract}

Keywords: metabolic syndrome; randomized controlled dietary intervention; gene expression; peripheral blood mononuclear cells; inflammation

\title{
1. Introduction
}

The metabolic syndrome (MetS) includes a cluster of related risk factors causing increased risk of cardiovascular diseases (CVD) and type 2 diabetes mellitus (T2DM). Central obesity is one of the major factors causing MetS, and metabolic alterations caused by obesity are associated with low-grade chronic inflammation [1-3]. The development of MetS is associated with a sedentary lifestyle, excessive energy intake, and an unhealthy diet [4]. It is well known that a Mediterranean-style dietary pattern reduces the risk of MetS [4,5]. The biological mechanisms causing the beneficial effects of a healthy diet are, however, largely unknown.

Peripheral blood mononuclear cells (PBMCs) are immune cells consisting of lymphocytes and monocytes. It is well established that a number of dietary factors modulate gene expression profiles in PBMCs [6-15]. Since these cells play a key role in the process of inflammation and are exposed to many of the same circulating factors as organs and the arterial wall, they may provide information on how the diet influences systemic inflammation and metabolic changes in peripheral tissues [15].

We have previously shown that a Nordic diet (ND) improved the lipid profile, and the circulating inflammatory marker IL-1 receptor antagonist (IL-1Ra) in individuals with MetS compared to a control diet (CD) (The SYSDIET study) [16]. In addition, we have shown, using global transcriptome profiling, that an ND resulted in the differential expression of inflammatory gene pathways in subcutaneous adipose tissue and PBMCs compared with a CD [12,14]. The ND also resulted in the down-regulation of toll-like receptor 4 (TRL4), interleukin 18 (IL18), and thrombospondin (CD36), and the up-regulation of peroxisome proliferator-activated receptor delta (PPARD) after a $2 \mathrm{~h}$ oral glucose tolerance test in PBMCs [13].

In this sub-study of the SYSDIET study, our specific aim was to examine the effect of a ND compared to a $\mathrm{CD}$ on pre-determined inflammatory and lipid related gene transcripts in fasting PBMC samples. To further understand the mechanisms behind the improved lipid profile and the possible anti-inflammatory effect of ND, we hypothesized that the intake of an ND compared to a CD would alter the expression of inflammatory genes and genes involved in lipid metabolism. 


\section{Materials and Methods}

\subsection{The SYSDIET Study}

The SYSDIET study was a randomized controlled multi-center study conducted in 2009-2010 in Kuopio and Oulu (Finland), Uppsala and Lund (Sweden), Aarhus (Denmark) and Reykjavik (Iceland), as previously described [16]. The primary outcome was glucose tolerance and insulin sensitivity. The secondary outcomes were related to MetS risk factors, i.e., blood pressure, serum lipids, inflammatory markers and gene expression. The detailed information on the study design and the main measurements have been described previously [16]. Briefly, after a one-month run-in period, the participants were randomized into a CD group or an ND group for 18 to 24 weeks. The main inclusion criteria were a body mass index (BMI) of $27-38 \mathrm{~kg} / \mathrm{m}^{2}, 30-65$ years of age, and two other International Diabetes Federation (IDF) criteria for MetS [17]. A stable use of anti-hypertensive and lipid lowering medication during the intervention was allowed. The main exclusion criteria have been described previously [16].

The major visits were in the beginning ( 0 week), at 12 weeks, and at either 18 or 24 weeks (end of the study). The diets were isocaloric and the study participants were instructed to keep physical activity and body weight constant and their smoking and drinking habits or drug treatment during the study unchanged. All study participants provided written informed consent and local Ethical committees of all the centers included in the current analysis (Research Ethics Committee of the Hospital District of Northern Savo and Northern Ostrobothnia Hospital District, Oulu, Finland and Regional Ethical Review Board, Lund) approved the study protocol in accordance with the Helsinki Declaration. The study is registered at clinicaltrials.gov as NCT00992641.

For this sub-study of SYSDIET, we included participants $(\mathrm{n}=94)$ who had given PBMCs in Kuopio, Lund, and Oulu, and who fulfilled the inclusion criteria (Figure 1). In total, 54 participants in the ND group and 40 in the CD group were included, as previously reported by Leder et al. [13]. The maximum weight change during the study was $+/-4 \mathrm{~kg}$, none of the participants used statins, and the high-sensitivity C-reactive protein (hsCRP) was $<10 \mathrm{mg} / \mathrm{L}$ at baseline and at the end of the intervention, and the BMI was $<39 \mathrm{~kg} / \mathrm{m}^{2}$, as previously reported [13]. Two of the centers had 24 weeks of study length (Kuopio and Lund) and one center had 18 weeks of study length (Oulu) [16].

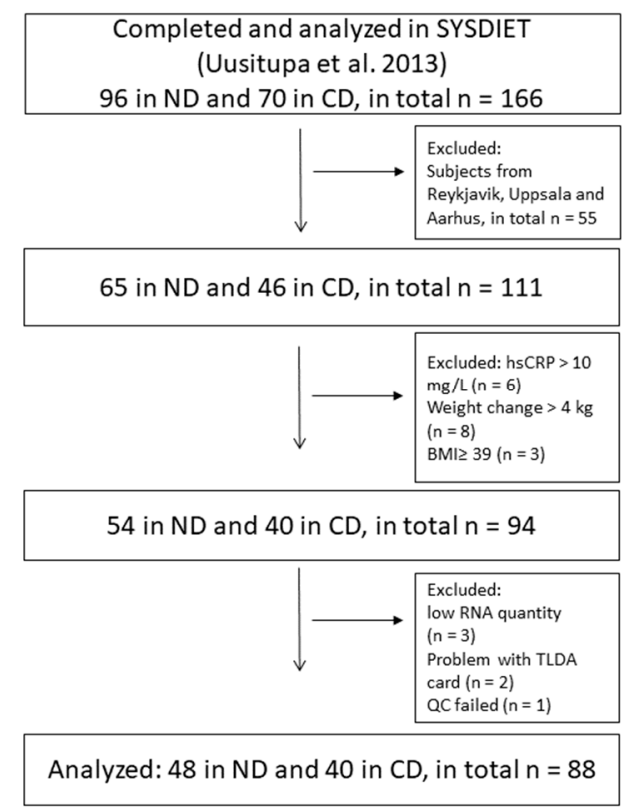

Figure 1. Flow chart of the participants. 


\subsection{Diet}

The Nordic nutrition recommendations formed the basis for the ND [18], and the mean nutrient intake in the Nordic countries formed the basis for the CD. The main emphasis in the ND group was whole-grain products, abundant use of berries, fruits and vegetables, rapeseed oil, three meals of fish per week, low-fat dairy products and the avoidance of sugar-sweetened products. More details about the diet is described elsewhere [16]. To assess the dietary intake, the participants filled in a 4-day dietary record during the run-in period (baseline intake) and three times during the intervention period.

\subsection{Biochemical Measurements}

All standard laboratory measurements, and anthropometric measurements were performed locally according to the standard operational procedures [16]. The plasma interleukins, plasma tumor necrosis factor receptor II (TNF RII), and serum high molecular weight (HMW) adiponectin were measured using ELISA, as previously described [16].

\subsection{Sampling of PBMCs and RNA Extraction}

The PBMCs were isolated from blood samples collected after overnight fasting (12 h) using cell preparation tubes (CPT) according to the manufacturer's instructions (Becton, Dickinson and Company, Franklin Lakes, NJ, USA) within 30 to $45 \mathrm{~min}$. All PBMC samples for the RNA analyses were prepared in the same laboratory (Karolinska Institute, Stockholm). The total RNA was extracted using the RNeasy Mini Kit according to the manufacturer's instructions (Qiagen, Valencia, CA, USA). The RNA integrity was checked using a Bioanalyzer device (Agilent 2100 Bioanalyzer, Agilent Technologies, Santa Clara, CA, USA).

\subsection{Real-Time Polymerase Chain Reaction RT-qPCR}

The RNA from all samples was reverse transcribed by a high-capacity cDNA reverse transcription kit (Applied Biosystems, Foster City, CA, USA). The selection of genes was primarily based on previous dietary intervention studies where the PBMC gene expression of lipid and cholesterol metabolism genes was modulated.

RT-qPCR was performed on an ABI PRISM 7900HT (Applied Biosystems). TaqMan Array Micro Fluidic Cards (Applied Biosystems) were used for RT-qPCR amplification of the gene transcripts. Three samples were excluded due to a low RNA quality, two samples were excluded due to technical problems, and one samples was excluded after quality control. In total, 88 samples were included in the final analyses. $\Delta \mathrm{Ct}$ was calculated as $\mathrm{Ct}_{\text {(reference gene) }}-\mathrm{Ct}_{\text {(target) }}$ and the $\log$ ratio $(\Delta \Delta \mathrm{Ct})$ was calculated as $\Delta \mathrm{Ct}_{\text {(end of study) }}-\Delta \mathrm{Ct}_{\text {(baseline) }}$. The TATA-binding protein $(T B P)$ was selected as the reference gene for normalization.

\subsection{Statistical Analysis}

Gene expression (Ct-values) was normalized using TBP as a reference gene, and the change from the baseline to the end of study was calculated as a log ratio (delta $\mathrm{Ct}$ (end of study) - delta Ct (baseline)). The difference between the CD and the ND was tested with a linear regression model, adjusted for age, sex and study center. The differences between baseline and end-of-study gene expression within the groups were tested with a paired $t$-test. The correlations between gene expression changes and changes in various biochemical measures and inflammatory markers were analyzed with Spearman's correlation using the $r c o r r$ function. $p$-values $<0.05$ were considered significant. All statistical analyses were performed in $\mathrm{R}$. 


\section{Results}

\subsection{Baseline Characteristics}

Eighty-eight individuals ( $\mathrm{n}=48 \mathrm{ND}$ group, $\mathrm{n}=40 \mathrm{CD}$ group) were included in the analyses of the present study (Figure 1). Their baseline characteristics are shown in Table 1.

Table 1. Baseline characteristics of the participants.

\begin{tabular}{ccccc}
\hline & \multicolumn{2}{c}{ CD (n= 40) } & \multicolumn{2}{c}{ ND (n = 48) } \\
\hline Male $(\mathrm{n}, \%)$ & 15 & $(37.5 \%)$ & 15 & $(31.3 \%)$ \\
Age $($ years) & 55.8 & $(7.8)$ & 54.2 & $(8.3)$ \\
BMI $\left(\mathrm{kg} / \mathrm{m}^{2}\right)$ & 31.9 & $(2.7)$ & 31.7 & $(3.1)$ \\
Waist circumference $(\mathrm{cm})$ & 105.4 & $(9.3)$ & 102.6 & $(9.0)$ \\
BP systolic $(\mathrm{mmHg})$ & 131 & $(17)$ & 127 & $(14)$ \\
BP diastolic $(\mathrm{mmHg})$ & 82 & $(12)$ & 83 & $(10)$ \\
Glucose $(\mathrm{mmol} / \mathrm{L})$ & 5.8 & $(0.6)$ & 5.9 & $(0.6)$ \\
Insulin $(\mathrm{pmol} / \mathrm{L})$ & 59.5 & $(47-80.8)$ & 56.0 & $(41.8-75.3)$ \\
Triglycerides $(\mathrm{mmol} / \mathrm{L})$ & 1.5 & $(0.5)$ & 1.5 & $(0.7)$ \\
Total cholesterol $(\mathrm{mmol} / \mathrm{L})$ & 5.3 & $(1)$ & 5.3 & $(1)$ \\
HDL cholesterol $(\mathrm{mmol} / \mathrm{L})$ & 1.3 & $(0.5)$ & 1.4 & $(0.3)$ \\
LDL cholesterol $(\mathrm{mmol} / \mathrm{L})$ & 3.3 & $(0.9)$ & 3.2 & $(0.9)$ \\
hsCRP $(\mathrm{mg} / \mathrm{L})$ & 1.5 & $(0.9-3.1)$ & 1.5 & $(0.8-2.8)$ \\
sTNFRII $(\mathrm{ng} / \mathrm{L})$ & 1900 & $(415)$ & 1953 & $(466)$ \\
IL-6 $(\mathrm{ng} / \mathrm{L})$ & 1.3 & $(1.1-1.7)$ & 1.3 & $(1-1.8)$ \\
IL-10 $(\mathrm{ng} / \mathrm{L})$ & 0.9 & $(0.8-1.5)$ & 0.8 & $(0.8-1.5)$ \\
IL-1 $\beta(\mathrm{ng} / \mathrm{L})$ & 0.12 & $(0.12-0.17)$ & 0.12 & $(0.12-0.13)$ \\
IL1 Ra $(\mathrm{ng} / \mathrm{L})$ & 309 & $(238-463)$ & 301 & $(220-466)$ \\
HMW adiponectin $(\mu \mathrm{gg} / \mathrm{L})$ & 3.6 & $(2.2-6.7)$ & 3.9 & $(2.8-6)$ \\
\hline Values are presented either as mean (SD), median $(25$ th-75th percentile) or n (\%).
\end{tabular}

The changes in nutrient intake were in agreement with the results obtained for the whole study population, as previously reported [13]. The polyunsaturated fatty acid (PUFA) intake increased, saturated fatty acid (SFA) intake decreased and the intakes of $\beta$-carotene and fiber increased in the ND group compared to the CD group.

\subsection{Gene Expression Profiling in PBMCs}

Compared to the CD group, the expression level of TNFRSF1A was significantly down-regulated in the ND group after intervention $(p=0.004)$, whereas the expression level of RELA was significantly increased ( $p=0.016$ ) after the intervention (Figure 2). No other differences in gene expression among inflammatory genes were observed between the groups after the intervention (Figure 3, Supplementary Table S1). No altered expression levels of the lipid metabolism-related genes were observed in the ND group compared to the CD group (Figure 4, Supplementary Table S1).

A within-group analysis showed that the between-group differences were mediated by down-regulation of the TNFRSF1A gene in the ND group ( $p=0.037)$ (Supplementary Figure S1), whereas the expression level of the RELA gene was significantly down-regulated in the CD group $(p=0.007)$ (Supplementary Figure S2). In addition, there were several within-group changes in both the ND and the CD groups (Supplementary Figures S1 and S2). 


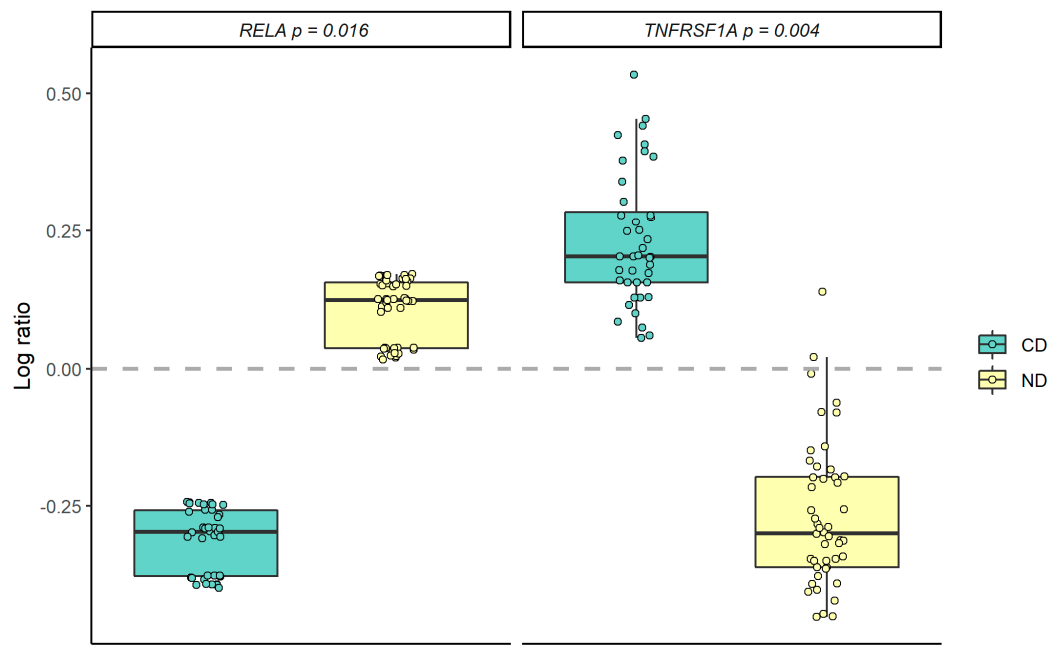

Figure 2. Gene expression changes (log ratio) of RELA and TNFRSF1A in the Nordic diet (ND) and control diet (CD) groups, adjusted for age, sex and study center. $\Delta \mathrm{Ct}$ was calculated as $\mathrm{Ct}_{\text {(reference gene) }}$ $\mathrm{Ct}_{\text {(target) }}$, and the log ratio $(\Delta \Delta \mathrm{Ct})$ was calculated as $\Delta \mathrm{Ct}_{\text {(end of study) }}-\Delta \mathrm{Ct}_{\text {(baseline) }}$. Differences between the groups were tested with a linear regression model. $p$-values $<0.05$ were considered significant.

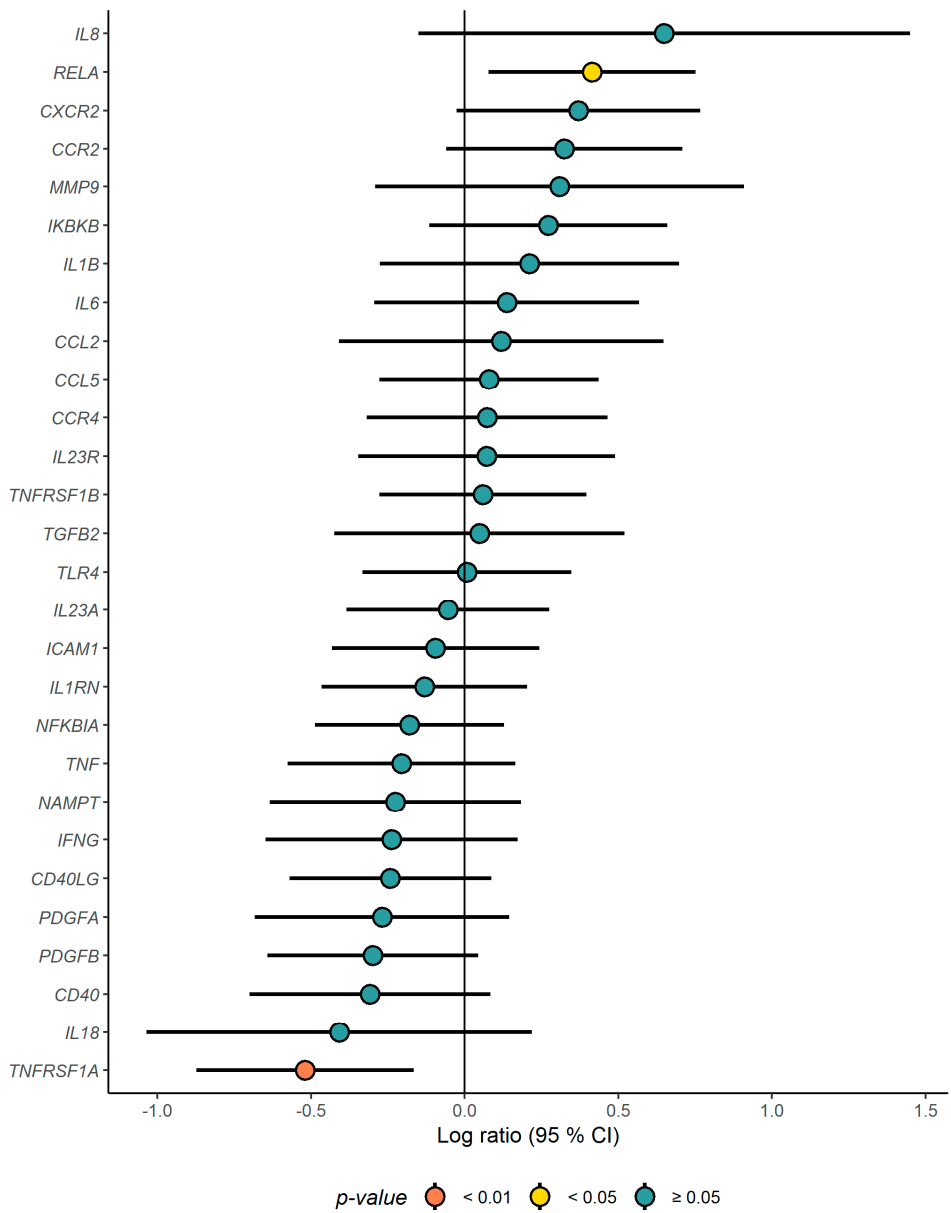

Figure 3. Gene expression changes (log ratio) in the ND relative to the CD of inflammation related genes. $\Delta \mathrm{Ct}$ was calculated as $\mathrm{Ct}_{\text {(reference gene) }}-\mathrm{Ct}_{\text {(target) }}$, and the $\log$ ratio $(\Delta \Delta \mathrm{Ct})$ was calculated as $\Delta \mathrm{Ct}_{\text {(end of study) }}-\Delta \mathrm{Ct}_{\text {(baseline) }}$. Differences between the groups were tested with a linear regression model, adjusted for age, sex and study center. $p$-values $<0.05$ were considered significant. 


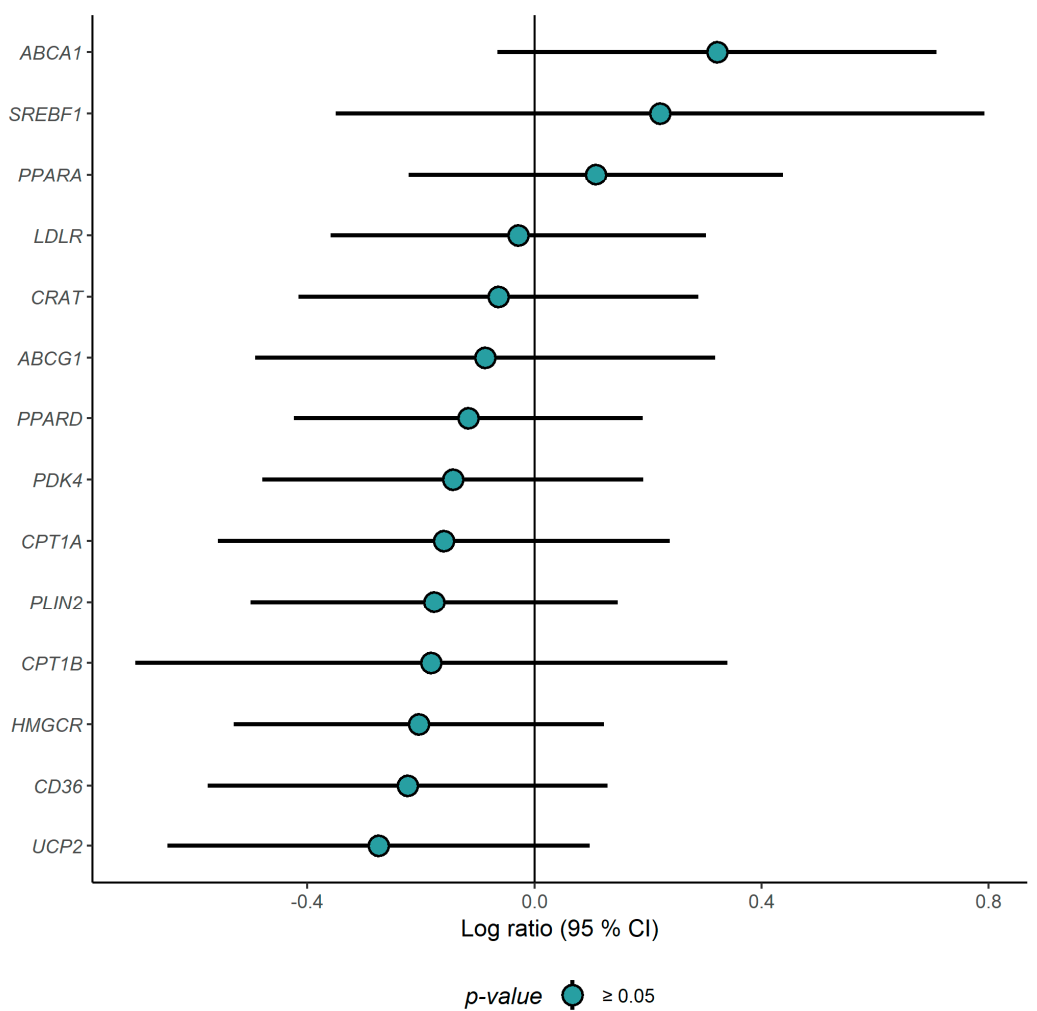

Figure 4. Gene expression changes (log ratio) in the ND relative to the CD of lipid metabolism-related genes. $\Delta \mathrm{Ct}$ was calculated as $\mathrm{Ct}_{\text {(reference gene) }}-\mathrm{Ct}_{\text {(target) }}$, and the $\log$ ratio $(\Delta \Delta \mathrm{Ct})$ was calculated as $\Delta \mathrm{Ct}_{(\mathrm{end} \text { of study) }}-\Delta \mathrm{Ct}_{(\text {baseline })}$. Differences between the groups were tested with a linear regression model, adjusted for age, sex and study center. $p$-values $<0.05$ were considered significant.

\subsection{Correlation Analysis}

We correlated the changes in gene expression of TNFRSF1A and RELA with changes in several plasma markers related to health, irrespective of group. Whereas the change in the mRNA level of TNFRSF1A did not significantly correlate with any of the circulating metabolites, the change in the mRNA level of RELA positively correlated with the change in hsCRP concentration and negatively with the change in low-density lipoprotein (LDL)-cholesterol (LDL-C) concentration, respectively (data not shown).

\section{Discussion}

In the present study, we investigated the impact of a ND compared to a CD on inflammation and lipid metabolism-related genes of PBMCs in individuals with MetS participating in a multi-center intervention study for 18/24 weeks. The ND group had an increased expression level of RELA and a decreased expression level of TNFRSF1A in their isolated PBMCs compared to the CD group. Our data are in line with previous findings [19] where the expression level of several inflammatory genes such as TNFRSF1A and TNFRSF1B were down-regulated in PBMCs in a diet-induced weight loss study including 34 overweight individuals with abnormal glucose metabolism and MetS. In the present study, the individuals kept a stable body weight, but despite this, the ND down-regulated the gene expression level of TNFRSF1A, which supports the key role of diet in the treatment of the MetS. Our data also agree with, and extend our previous findings using a transcriptome-wide approach, demonstrating that pathways regulating the mitochondrial electron transport chain, immune response, and cell cycle in addition to gene transcripts with common motifs for the transcription factors Nuclear respiratory factor 1 (NRF1), NRF2, and nuclear factor kappa-light-chain-enhancer of activated B cells (NF- $\mathrm{KB}$ ) were down-regulated in the ND group compared to CD [14]. 
The increased intake of SFA in contrast to PUFA has been shown to increase liver fat and liver enzyme levels [20-22]. While the individuals included in the present study were characterized by having MetS, studies have suggested that both MetS and non-alcoholic fatty liver disease (NAFLD) very early in the progression share several common stimulatory mechanisms [23]. Interestingly, the pro-inflammatory TNF superfamily has been suggested to play a key role in the development of NAFLD and subsequently non-alcoholic steatohepatitis (NASH) [24]. Increased gene expression levels of both TNF and TNFR1 have been shown in humans with NASH, thus supporting a role for the TNFR1 pathway in the progression of NASH. Our finding of a reduced gene expression level of TNFRSF1A could thus be associated with attenuation of both inflammation and liver fat accumulation. The inflammatory gene expression profile in PBMCs has previously been shown to reflect the immune component of white adipose tissue [25] and liver lipid metabolism [26], further supporting the notion that PBMC gene expression may be useful in providing information about metabolic health in general.

In addition, the gene transcript RELA encoding for the p65 NF- $\mathrm{KB}$ subunit was significantly up-regulated in the ND group compared to the CD group. This between-group difference was primarily caused by a down-regulation of this gene within the CD group and therefore, this finding may suggest that the ND plays a role in maintaining homeostasis in the immune response. The activation of the NF- $\mathrm{kB}$ system seems to be important in promoting liver inflammation [27]; however, the role of RELA (p65) in the development of atherosclerosis is less clear. Nonetheless, RELA has been shown to be a key regulator of the inflammatory response in macrophages [28], where overexpression of RELA in apolipoprotein $E$ knock-out mice led to reduced atherosclerotic lesion size and higher energy expenditure. Thus, the effects of the activation of RELA and the NFKB pathway may vary in different tissues (liver/macrophages) and depend on the inflammatory state of the individuals (chronic/acute). The observed increase in RELA gene expression in PBMCs (precursor for macrophages) in the present study in the ND group compared with the CD group in the present study may, thus, be associated with an atheroprotective effect. However, future studies are warranted to further understand the role of an ND in the regulation of RELA, and the consequences of this regulation in immune cells.

None of the gene transcripts encoding proteins related to lipid metabolism were changed by the ND compared to the CD. We have previously shown that exchanging SFA with PUFA reduced total cholesterol and LDL-C by $9 \%$ and $11 \%$, respectively, among subjects with slightly elevated cholesterol [29], and changed the PBMC expression levels of several lipid metabolism-related genes [30]. The reason why we did not observe any changes in lipid metabolism-related genes in the present study, may be due to the smaller effect of the ND on the lipid profile as previously shown [16].

Interestingly, when only comparing changes in gene expression levels within the groups, we observed that there are many more genes regulated within the CD group compared to within the ND group. We can speculate that the reason for the larger effect in the CD group may be that volunteers in this kind of study are usually a health-conscious population. If the subjects in the CD group changed to a not-so-healthy diet, a more pronounced effect on the gene expression profiles might be seen. An unhealthy diet may stress the system more than eating a healthier diet (ND group). These findings are also in line with the results from the white adipose tissue whole genome expression profiles in the SYSDIET study [12].

A major strength of the present study is that we were able to use data from a well-designed randomized controlled dietary intervention study, where the changes in gene expression in the ND group were compared to the changes in a CD group. A problem with longitudinal studies using PBMCs is the short (2-3 weeks) life span of the mononuclear cells. Thus, some cells were exposed to the diet longer than other cells, which might influence the results.

\section{Conclusions}

We found an increased expression of RELA and a reduced expression level of TNFRSF1A in individuals with MetS after the intervention. These data provide further evidence that the consumption of a ND compared to a CD may affect the immune response at the molecular level in PBMCs. Within 
the preselected genes that were examined, no gene expression changes in lipid metabolism-related genes were observed between the ND and $C D$, and further studies are needed to confirm if PBMC gene expression profiles may explain the improvement of the lipid profile in circulation observed after intake of a ND.

Supplementary Materials: The following are available online at http://www.mdpi.com/2072-6643/11/12/2932/s1, Figure S1. Gene expression changes (deltaCt) in the ND group at baseline and end of study. $\Delta \mathrm{Ct}$ was calculated as $\mathrm{Ct}_{\text {(reference gene) }}-\mathrm{Ct}_{\text {(target) }}$, and the $\log$ ratio $(\Delta \Delta \mathrm{Ct})$ was calculated as $\Delta \mathrm{Ct}_{\text {(end of study) }}-\Delta \mathrm{Ct}_{\text {(baseline) }}$. Differences between the time points are tested with a paired $t$-test. $p$-values $<0.05$ were considered significant. Figure S2. Gene expression changes (deltaCt) in the $\mathrm{CD}$ group at baseline and end of study. $\Delta \mathrm{Ct}$ was calculated as $\mathrm{Ct}_{\text {(reference gene) }}-\mathrm{Ct}_{\text {(target) }}$, and the $\log$ ratio $(\Delta \Delta \mathrm{Ct})$ was calculated as $\Delta \mathrm{Ct}_{\text {(end of study) }}-\Delta \mathrm{Ct}_{\text {(baseline) }}$. Differences between the time points are tested with a paired $t$-test. $p$-values $<0.05$ were considered significant. Table S1. Gene expression change from baseline to end of study in SYSDIET relative to control group,

Author Contributions: S.M.U., K.B.H., M.C.W.M., L.L., V.D.d.M., I.D., U.S., C.C., K.H., L.O.D., I.G., L.C., B.Å., K.-H.H., M.J.S., U.R., I.T., K.S.P., P.A., M.U., and M.K.: designed the research; S.M.U., K.B.H., M.C.W.M., L.L., I.D., U.S., J.P., K.H., I.G., L.C., B.Å., F.R., J.H., K.-H.H., M.J.S., U.R., I.T., K.S.P., P.A., M.U., M.K.: conducted the research; S.M.U., K.B.H., A.R., M.C.W.M., L.L., M.K.: analyzed the data or performed statistical analysis; S.M.U., K.B.H., AR, M.C.W.M., M.K.: wrote the first draft of the manuscript and had primary responsibility for the final content; M.K., K.S.P., M.U.: were responsible for the coordination of the SYSDIET consortium. All authors have participated in and critically reviewed the manuscript and accepted it to be submitted.

Funding: This research was funded by the NordForsk Nordic Centre of Excellence in Food, Nutrition and Health project 070014 [SYSDIET (Systems Biology in Controlled Dietary Interventions and Cohort Studies)] and further, the University of Oslo (Norway), Throne Holst Foundation (Norway), Academy of Finland, Swedish Research council, Svenska Diabetesförbundet, SRP Diabetes, Finnish Diabetes Research Foundation, Finnish Foundation for Cardiovascular Research, the Sigrid Juselius Foundation, EVO funding from Kuopio University Hospital (Finland), the Druvan Foundation, Skåne University Hospital, the Heart-Lung Foundation, Diabetesfonden and Foundation Cerealia (Sweden), the Agricultural Productivity Fund, and the Research Fund of the University of Iceland (Iceland).

Acknowledgments: We thank Maritta Siloaho for their excellent expertise and advice for biochemical measurements and Marika Rönnholm for their excellent technical assistance.

Conflicts of Interest: Ulven has received research grants from Tine DA, Mills DA, and Olympic Seafood, none of which are related to the content of this manuscript. Holven has received research grants and/or personal fees from Tine DA, Mills DA, Olympic Seafood, Amgen, Kaneka, Sanofi, and Pronova, none of which are related to the content of this manuscript. The other authors declare no conflict of interest.

\section{References}

1. Wellen, K.E.; Hotamisligil, G.S. Inflammation, stress, and diabetes. J. Clin. Investig. 2005, 115, 1111-1119. [CrossRef] [PubMed]

2. Wellen, K.E.; Hotamisligil, G.S. Obesity-induced inflammatory changes in adipose tissue. J. Clin. Investig. 2003, 112, 1785-1788. [CrossRef] [PubMed]

3. Bastard, J.P.; Maachi, M.; Lagathu, C.; Kim, M.J.; Caron, M.; Vidal, H.; Capeau, J.; Feve, B. Recent advances in the relationship between obesity, inflammation, and insulin resistance. Eur. Cytokine Netw. 2006, 17, 4-12. [PubMed]

4. Panagiotakos, D.B.; Pitsavos, C.; Chrysohoou, C.; Skoumas, J.; Tousoulis, D.; Toutouza, M.; Toutouzas, P.; Stefanadis, C. Impact of lifestyle habits on the prevalence of the metabolic syndrome among Greek adults from the ATTICA study. Am. Heart J. 2004, 147, 106-112. [CrossRef]

5. Esposito, K.; Marfella, R.; Ciotola, M.; Di Palo, C.; Giugliano, F.; Giugliano, G.; D'Armiento, M.; D’Andrea, F.; Giugliano, D. Effect of a mediterranean-style diet on endothelial dysfunction and markers of vascular inflammation in the metabolic syndrome: A randomized trial. JAMA 2004, 292, 1440-1446. [CrossRef]

6. De Mello, V.D.; Erkkilä, A.T.; Schwab, U.S.; Pulkkinen, L.; Kolehmainen, M.; Atalay, M.; Mussalo, H.; Lankinen, M.; Orešič, M.; Lehto, S.; et al. The effect of fatty or lean fish intake on inflammatory gene expression in peripheral blood mononuclear cells of patients with coronary heart disease. Eur. J. Nutr. 2009, 48, 447-455. [CrossRef]

7. van Dijk, S.J.; Feskens, E.J.; Bos, M.B.; de Groot, L.C.; de Vries, J.H.; Müller, M.; Afman, L.A. Consumption of a high monounsaturated fat diet reduces oxidative phosphorylation gene expression in peripheral blood mononuclear cells of abdominally overweight men and women. J. Nutr. 2012, 142, 1219-1225. [CrossRef] 
8. Myhrstad, M.C.W.; Ulven, S.M.; Günther, C.C.; Ottestad, I.; Holden, M.; Ryeng, E.; Borge, G.I.; Kohler, A.; Brønner, K.W.; Thoresen, M.; et al. Fish oil supplementation induces expression of genes related to cell cycle, endoplasmic reticulum stress and apoptosis in peripheral blood mononuclear cells: A transcriptomic approach. J. Intern. Med. 2014, 276, 498-511. [CrossRef]

9. Radler, U.; Stangl, H.; Lechner, S.; Lienbacher, G.; Krepp, R.; Zeller, E.; Brachinger, M.; Eller-Berndl, D.; Fischer, A.; Anzur, C.; et al. A combination of (omega-3) polyunsaturated fatty acids, polyphenols and L-carnitine reduces the plasma lipid levels and increases the expression of genes involved in fatty acid oxidation in human peripheral blood mononuclear cells and HepG2 cells. Ann. Nutr. Metab. 2011, 58, 133-140. [CrossRef]

10. Afman, L.; Milenkovic, D.; Roche, H.M. Nutritional aspects of metabolic inflammation in relation to health-insights from transcriptomic biomarkers in PBMC of fatty acids and polyphenols. Mol. Nutr. food Res. 2014, 58, 1708-1720. [CrossRef]

11. Kolehmainen, M.; Mykkänen, O.; Kirjavainen, P.V.; Leppänen, T.; Moilanen, E.; Adriaens, M.; Laaksonen, D.E.; Hallikainen, M.; Pimiä, P.R.; Pulkkinen, L.; et al. Bilberries reduce low-grade inflammation in individuals with features of metabolic syndrome. Mol. Nutr. Food Res. 2012, 56, 1501-1510. [CrossRef] [PubMed]

12. Kolehmainen, M.; Ulven, S.M.; Paananen, J.; de Mello, V.; Schwab, U.; Carlberg, C.; Myhrstad, M.; Pihlajamäki, J.; Dungner, E.; Sjölin, E.; et al. Healthy Nordic diet downregulates the expression of genes involved in inflammation in subcutaneous adipose tissue in individuals with features of the metabolic syndrome. Am. J. Clin. Nutr. 2015, 101, 228-239. [CrossRef] [PubMed]

13. Leder, L.; Kolehmainen, M.; Narverud, I.; Dahlman, I.; Myhrstad, M.C.; De Mello, V.D.; Paananen, J.; Carlberg, C.; Schwab, U.; Herzig, K.-H.; et al. Effects of a healthy Nordic diet on gene expression changes in peripheral blood mononuclear cells in response to an oral glucose tolerance test in subjects with metabolic syndrome: A SYSDIET sub-study. Genes Nutr. 2016, 11, 3. [CrossRef] [PubMed]

14. Myhrstad, M.C.; de Mello, V.D.; Dahlman, I.; Kolehmainen, M.; Paananen, J.; Rundblad, A.; Carlberg, C.; Olstad, O.K.; Pihlajamäki, J.; Holven, K.B.; et al. Healthy Nordic Diet Modulates the Expression of Genes Related to Mitochondrial Function and Immune Response in Peripheral Blood Mononuclear Cells from Subjects with Metabolic Syndrome-A SYSDIET Sub-Study. Mol. Nutr. Food Res. 2019, 68, 1801405. [CrossRef]

15. de Mello, V.D.; Kolehmanien, M.; Schwab, U.; Pulkkinen, L.; Uusitupa, M. Gene expression of peripheral blood mononuclear cells as a tool in dietary intervention studies: What do we know so far? Mol. Nutr. Food Res. 2012, 56, 1160-1172. [CrossRef]

16. Uusitupa, M.; Hermansen, K.; Savolainen, M.J.; Schwab, U.; Kolehmainen, M.; Brader, L.; Mortensen, L.S.; Cloetens, L.; Persson, A.J.; Önning, G.; et al. Effects of an isocaloric healthy Nordic diet on insulin sensitivity, lipid profile and inflammation markers in metabolic syndrome-A randomized study (SYSDIET). J. Intern. Med. 2013, 274, 52-66. [CrossRef]

17. Alberti, K.G.; Eckel, R.H.; Grundy, S.M.; Zimmet, P.Z.; Cleeman, J.I.; Donato, K.A.; Fruchart, J.C.; James, W.P.T.; Loria, C.M.; Smith, S.C., Jr. Harmonizing the metabolic syndrome: A joint interim statement of the International Diabetes Federation Task Force on Epidemiology and Prevention; National Heart, Lung, and Blood Institute; American Heart Association; World Heart Federation; International Atherosclerosis Society; and International Association for the Study of Obesity. Circulation 2009, 120, 1640-1645.

18. Nordic Council of Ministers. Nordic Nutrition Recommendations 2012: Intergrating Nutrition and Physical Activity; Nordic Council of Ministers: Copenhagen, Denmark, 2014.

19. De Mello, V.D.F.; Kolehmainen, M.; Pulkkinen, L.; Schwab, U.; Mager, U.; Laaksonen, D.E.; Gylling, H.; Atalay, M.; Rauramaa, R.; Uusitupa, M. Downregulation of genes involved in NFkappaB activation in peripheral blood mononuclear cells after weight loss is associated with the improvement of insulin sensitivity in individuals with the metabolic syndrome: The GENOBIN study. Diabetologia 2008, 51, 2060-2067. [CrossRef]

20. Rosqvist, F.; Iggman, D.; Kullberg, J.; Cedernaes, J.; Johansson, H.E.; Larsson, A. Overfeeding polyunsaturated and saturated fat causes distinct effects on liver and visceral fat accumulation in humans. Diabetes 2014, 63, 2356-2368. [CrossRef]

21. Rosqvist, F.; Kullberg, J.; Ståhlman, M.; Cedernaes, J.; Heurling, K.; Johansson, H.E.; Iggman, D.; Wilking, H.; Larsson, A.; Eriksson, O.; et al. Overeating saturated fat promotes fatty liver and ceramides compared to polyunsaturated fat: A randomized trial. J. Clin. Endocrinol. Metab. 2019, 104, 6207-6219. [CrossRef] 
22. Bjermo, H.; Iggman, D.; Kullberg, J.; Dahlman, I.; Johansson, L.; Persson, L.; Berglund, J.; Pulkki, K.; Basu, S.; Uusitupa, M.; et al. Effects of n-6 PUFAs compared with SFAs on liver fat, lipoproteins, and inflammation in abdominal obesity: A randomized controlled trial. Am. J. Clin. Nutr. 2012, 95, 1003-1012. [CrossRef] [PubMed]

23. Preuss, H.G.; Kaats, G.R.; Mrvichin, N.; Swaroop, A.; Bagchi, D.; Clouatre, D. Examining the Relationship Between Nonalcoholic Fatty Liver Disease and the Metabolic Syndrome in Nondiabetic Subjects. J. Am. Coll. Nutr. 2018, 37, 457-465. [CrossRef] [PubMed]

24. Aparicio-Vergara, M.; Hommelberg, P.P.; Schreurs, M.; Gruben, N.; Stienstra, R.; Shiri-Sverdlov, R.; Kloosterhuis, N.J.; de Bruin, A.; van de Sluis, B.; Koonen, D.P.Y.; et al. Tumor necrosis factor receptor 1 gain-of-function mutation aggravates nonalcoholic fatty liver disease but does not cause insulin resistance in a murine model. Hepatology 2013, 57, 566-576. [CrossRef] [PubMed]

25. O'Grada, C.M.; Morine, M.J.; Morris, C.; Ryan, M.; Dillon, E.T.; Walsh, M.; Gibney, R.R.; Brennan, L.; Gibney, M.J.; Roche, H.M. PBMCs reflect the immune component of the WAT transcriptome-Implications as biomarkers of metabolic health in the postprandial state. Mol. Nutr. Food Res. 2014, 58, 808-820. [CrossRef]

26. Bouwens, M.; Afman, L.A.; Müller, M. Fasting induces changes in peripheral blood mononuclear cell gene expression profiles related to increases in fatty acid beta-oxidation: Functional role of peroxisome proliferator activated receptor alpha in human peripheral blood mononuclear cells. Am. J. Clin. Nutr. 2007, 86, 1515-1523. [CrossRef]

27. Li, J.; Sapper, T.N.; Mah, E.; Moller, M.V.; Kim, J.B.; Chitchumroonchokchai, C. Green tea extract treatment reduces NFkappaB activation in mice with diet-induced nonalcoholic steatohepatitis by lowering TNFR1 and TLR4 expression and ligand availability. J. Nutr. Biochem. 2017, 41, 34-41. [CrossRef]

28. Ye, X.; Jiang, X.; Guo, W.; Clark, K.; Gao, Z. Overexpression of NF-kappaB p65 in macrophages ameliorates atherosclerosis in apoE-knockout mice. Am. J. Physiol. Endocrinol. Metab. 2013, 305, E1375-E1383. [CrossRef]

29. Ulven, S.M.; Leder, L.; Elind, E.; Ottestad, I.; Christensen, J.J.; Telle-Hansen, V.H. Exchanging a few commercial, regularly consumed food items with improved fat quality reduces total cholesterol and LDL-cholesterol: A double-blind, randomised controlled trial. Br. J. Nutr. 2016, 116, 1383-1393. [CrossRef]

30. Ulven, S.M.; Christensen, J.J.; Nygård, O.; Svardal, A.; Leder, L.; Ottestad, I. Using metabolic profiling and gene expression analyses to explore molecular effects of replacing saturated fat with polyunsaturated fat-a randomized controlled dietary intervention study. Am. J. Clin. Nutr. 2019, 109, 1239-1250. [CrossRef] 